\title{
A New Paradigm for Diabetes and Obesity: the Hepatic Insulin Sensitizing Substance (HISS) Hypothesis
}

\author{
W. Wayne Lautt ${ }^{1} *$ \\ ${ }^{1}$ Department of Pharmacology \& Therapeutics, Faculty of Medicine, University of Manitoba, \\ Winnipeg, Manitoba R3E 0T6, Canada
}

Received March 11, 2004

\begin{abstract}
The glucose disposal effect of insulin after a meal is accounted for in approximately equal measure by the direct action of insulin and the action of HISS (hepatic insulin sensitizing substance) released from the liver and acting on skeletal muscle to stimulate glucose storage as glycogen. The ability of insulin to cause HISS release is determined by hepatic parasympathetic nerves. Eliminating the parasympathetic signal by surgical denervation of the liver or by blockade of hepatic muscarinic receptors, hepatic nitric oxide synthase, or hepatic cyclooxygenase results in insulin resistance that can be accounted for by the absence of HISS action and is referred to as HISS-dependent insulin resistance (HDIR). Animal models in which the insulin resistance has been shown to be HDIR includes the spontaneously hypertensive rat, sucrose fed rats, animals with liver disease, adult offspring of fetal alcohol exposure, acute stress, and ageing. We suggest that HDIR accounts for the major metabolic disturbances in type 2 diabetes, including the postprandial hyperglycemia that results in the majority of pathologies related to diabetes. The observation of meal-induced insulin sensitization (MIS) and the role of HISS allows for consideration of a new paradigm relating meal processing, diabetes, obesity, and insulin resistance. New diagnostic approaches and therapeutic targets are described.
\end{abstract}

Keywords: insulin resistance, obesity, hypertension, diabetes, feeding

\section{Introduction}

The objective of this review is to examine the broad implications of hepatic insulin sensitizing substance (HISS)-related glucose regulation. The "HISS hypothesis", first proposed in Germany in 1997, appears to explain a range of metabolic responses both in health and disease. The HISS hypothesis appears consistent with the following conclusions:

- HISS release is activated by insulin according to prandial status, maximal after feeding (shown).

- HISS is the primary stimulator of post-meal glucose storage as glycogen in skeletal muscle (shown).

- The most common forms of insulin resistance are accounted for by HISS-dependent insulin resistance (HDIR); an absence of HISS action (shown in

*Corresponding author. FAX: +1-204-975-7784

E-mail: wlautt@ms.umanitoba.ca

Invited article numerous animal models).

- HDIR accounts for "syndrome X" or the "metabolic syndrome" (hypothetical extension).

- HDIR accounts for postprandial hyperglycemia, hyperinsulinemia, and hyperlipidemia (not shown).

- HDIR results in a state of metabolic obesity with nutrients stored preferentially as fat (not shown).

- HDIR can be diagnosed, quantified, and treated (shown).

Although conclusions and speculations may be consistent with the hypothesis, this paradigm is at an early stage and many details will undoubtedly change. Speculation as to the missing links will hopefully be facilitated by this review of the current state of the HISS story.

\section{Overview of the HISS hypothesis (Fig. 1)}

In the fed state approximately $55 \%$ of the glucose disposal effect of insulin is accounted for by a meal- 


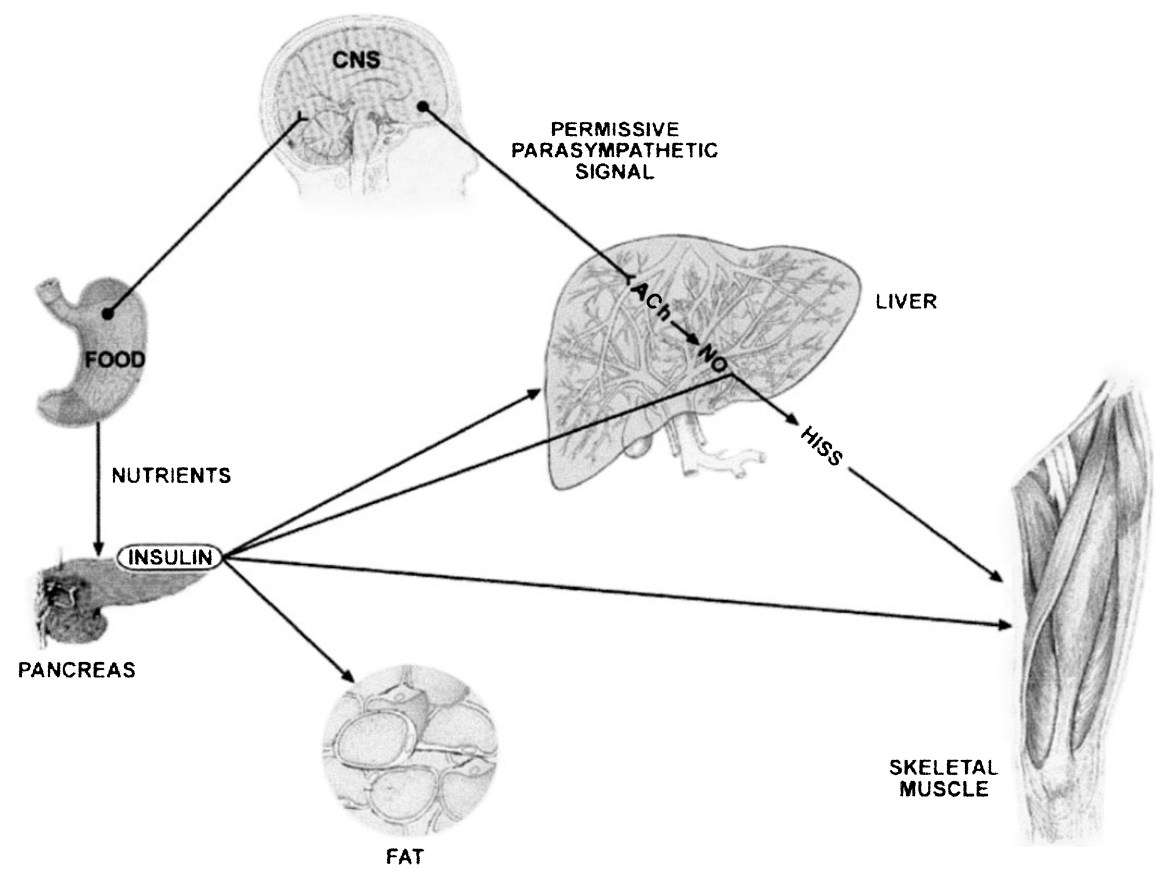

Fig. 1. Following a meal, insulin released by the pancreas results in stimulation of release of hepatic insulin sensitizing substance (HISS) from the liver. In order for insulin to produce HISS release, a parasympathetic permissive reflex that signals the presence of feeding must be present. HISS enters the bloodstream and stimulates the large skeletal muscle masses of the body to take up glucose and store it as glycogen. If the parasympathetic function is impaired, as it is in experimental models and many forms of disease states, HISS is not released after a meal thereby resulting in a greatly $(55 \%)$ diminished ability to store the glucose being absorbed from the meal. The response is hyperglycemia, hyperinsulinemia, and elevated production of fat. Elimination of the hepatic parasympathetic nerve function decreases the glucose disposal effect of insulin by $55 \%$ and results in HISS-dependent insulin resistance. The parasympathetic nerves work through activation of cholinergic muscarinic receptors in the liver and subsequent production of nitric oxide. Modified from a previously published figure in the Canadian Journal of Physiology and Pharmacology, volume 77, on page 555, 1999.

induced insulin sensitization (MIS) process whereby the response to insulin is doubled after a meal. Insulin results in release of a hormone from the liver that acts selectively on skeletal muscle to stimulate glucose uptake. This putative hormone is tentatively referred to as HISS. MIS decreases progressively with fasting, until after $24 \mathrm{~h}$ only the direct action of insulin is seen and a bolus of insulin is no longer capable of causing HISS release. MIS is regulated by a permissive signal from hepatic parasympathetic nerves in the liver that are activated soon after feeding and become progressively deactivated with the duration of fasting. The parasympathetic signal is regarded as permissive in that the signal, per se, does not result in the release of HISS or in notable effects on peripheral glucose metabolism. Rather, the background permissive signal allows pulses of insulin to result in insulin-dose-related pulsatile release of HISS.

The parasympathetic signal involves the release of acetylcholine that acts on hepatic muscarinic receptors, resulting in the production of nitric oxide (NO) and release of HISS. Hepatic glutathione levels, which are decreased by fasting and rapidly replenished by feeding, are also an integral part of the HISS regulatory pathway as is hepatic guanylyl cyclase. The cyclooxygenase (COX) pathway also appears to be involved with HISS regulation.

Blockade of the parasympathetic signal in fed animals results in a decrease of the glucose disposal effect of insulin by approximately $50 \%$ and reduces the insulin response to levels seen in the normal fasted state. HDIR is a physiological state produced by fasting and can be produced by any means that interrupts the parasympathetic signaling process including hepatic surgical denervation, blockade of hepatic muscarinic receptors or nitric oxide synthase (NOS), COX or adenylyl cyclase, or by depletion of hepatic glutathione levels. Pathophysiological conditions of a wide range result in HDIR. Animal models in which the insulin resistance has been shown to be HDIR includes the spontaneously hypertensive rat, sucrose fed rats, animals with liver disease induced by chronic bile duct ligation or chemical toxins, adult offspring of fetal alcohol exposure, acute stress, and ageing. 
The HISS hypothesis suggests that HDIR can account for the metabolic dysfunctions seen in the "metabolic syndrome" or "syndrome X". According to this hypothesis, HDIR in the fed state results in a severe state of insulin resistance that leads to postprandial hyperglycemia and a compensatory hyperinsulinemia with the increased insulin levels stimulating primarily adipose tissue and liver. The adipose tissue directly forms lipids; the limited glycogen storage capacity of the liver can rapidly become saturated with the result being that the remaining glucose uptake will result in the formation of lipids, thereby producing increased hepatic lipid concentration and increased circulating VLDL and hyperlipidemia.

HDIR can be diagnosed by determining insulin action before and after feeding. The HISS-independent component of insulin action (direct insulin effect) remains unaltered by fasting, atropine, or hepatic denervation. Chronic animal models of HDIR do not show notable alterations in HISS-independent insulin action. That is, the sensitivity to insulin, in the absence of HISS action, is not altered in most states of HDIR, including fasting. By the time diagnosis of type 2 diabetes is made using the traditional fasting state evaluations, the disease process has likely existed for years or even decades.

The ability to detect HDIR offers a potential powerful diagnostic tool. The ability to treat HDIR includes methods to mimic or potentiate the hepatic parasympathetic signal or elevate hepatic glutathione levels or various combinations of manipulation of the regulatory pathway. It is highly unlikely that HDIR in all the disease models described are produced by the same signaling defect, thus suggesting that a range of therapeutic approaches through regulation of HISS will lead to a new family of therapeutic compounds.

\section{Discovery of the role of HISS in MIS}

Although the dependence of whole body glucose disposal in response to insulin was first observed in my laboratory in 1991, the first publication implicating a regulatory role for hepatic parasympathetic nerves appeared in 1993 (1) and it was not until 1996 (2) that we first discovered that variability in permissive parasympathetic tone accounted for the variability in insulin sensitivity seen in randomly fed animals. This led us then to consider that the factor controlling the parasympathetic tone might be feeding. In 2001 (3), we first demonstrated that the HISS-dependent component of insulin action was regulated by the prandial status; HISS action accounted for approximately $55 \%$ of the response to insulin in animals that had recently eaten; HISS action was essentially absent after a 24-h fast. The HISS- independent component of insulin action is the response to administered insulin seen after blockade of HISS release (using surgical denervation of the liver or atropine to block the muscarinic receptors). The HISSindependent component of insulin action is not affected by feeding or fasting. The HISS-dependent component, however, progressively decreases to insignificance by a 24-h fast (3). Subsequent studies in conscious rats tested for insulin sensitivity in the fasted state confirmed that feeding resulted in a dramatic MIS that could be accounted for entirely by the HISS hypothesis (4).

An apparent anomaly is that HISS action has also been demonstrated in the fasted state in cats $(1,5)$ and dogs (6). However, these studies were capable of demonstrating HISS action probably because the duration of fasting was not sufficiently long to have eliminated the HISS component. Cats, rats, and dogs fasted for $16-18 \mathrm{~h}$ all show a HISS component that accounts for $25-35 \%$ of insulin action. Even a $24-h$ fast in rats will often show significant HISS action. The MIS process has also recently been demonstrated in mice (7) and humans (M.P. Macedo et al., unpublished observation) with the glucose disposal response to insulin more than doubling after a meal.

Terminology: New terminology was required for aspects of this paradigm. The term HISS (8) was introduced to describe a putative hormone released by the liver and acting on skeletal muscle. The meal-induced increase in glucose disposal seen in response to a bolus administration of insulin is accounted for by HISS. The MIS process is dependent upon hepatic release of HISS that acts on skeletal muscle. Because of the selective nature of HISS action on skeletal muscle but not liver or adipose tissue, it is clear that some unique substance is released by the liver. Thus, the word 'sensitization' does not refer to a true sensitization to the direct effect of insulin, which does not occur, but rather to a potentiation of the glucose disposal induced by administered insulin which is secondary to insulin-induced HISS release and subsequent HISS action. Similarly, MIS is meant to serve as a convenient term that describes the process by which feeding results in an increased metabolic response to insulin. HDIR describes the phenomenon whereby HISS release or action does not occur in response to insulin. The glucose disposal response to administered insulin that is eliminated by eliminating the effect of HISS is the HISS-dependent component of insulin action and the response remaining is the HISS-independent component of insulin action, probably representing the direct effect of insulin on insulin-sensitive tissues. 


\section{Pharmacodynamic studies of HISS}

It is possible to carry out detailed pharmacodynamic studies without the need to have the chemical identity of HISS as long as the biological activity of HISS can be accurately and precisely quantitated (9, reviewed). Although HISS action has been demonstrated through the use of arterial-venous glucose gradients across tissues $(6,10)$ and the insulin tolerance test $(1,11)$, early on we identified the need for a more appropriate tool for quantitating insulin action. The rapid insulin sensitivity test (RIST) is a rapidly sampled transient euglycemic clamp that determines glucose uptake stimulated by a bolus of insulin. A standard operating procedure for carrying out the RIST has been described (12, $13)$.

The RIST methodology: The operating procedure has previously been detailed (13). In order to obtain multiple blood samples without the need to flush catheters, a vascular shunt methodology was developed that involves inserting a catheter into the femoral artery and femoral vein with the connection between the two catheters consisting of a segment of silicone tubing that has a side catheter inserted to allow pressure monitoring. This shunt has also been used with catheters in the carotid artery and jugular vein, which is especially useful in experiments dealing with chronic cannulation and measurements in conscious rats (4). The vascular shunt allows for multiple arterial blood sampling by direct puncture into the silicone $\mathrm{A}-\mathrm{V}$ shunt. Pressure in the shunt is used to monitor patency of the shunt. A brief clamping of the venous end of the shunt allows determination of arterial pressure. Intravenous drug administration is made directly into the shunt. Briefly, the animals are allowed to stabilize for at least $30 \mathrm{~min}$, after the surgical preparation, prior to the first arterial glucose sampling. Arterial blood samples are then taken every $5 \mathrm{~min}$ by puncture from the shunt, and glucose concentrations are immediately analyzed by the oxidase method with a modified 1500 Sport Lactate Analyzer (Yellow Springs Instruments, Yellow Springs, CO, USA) until a stable basal glucose concentration is established. Insulin is then infused intravenously via the shunt at a dose of $50 \mathrm{mU} / \mathrm{kg}$ over a $5-\mathrm{min}$ period (in $0.5 \mathrm{ml}$ saline at $0.1 \mathrm{ml} / \mathrm{min}$ ). It can also be administered in a 30 -s bolus without significantly altering the outcome. To avoid hypoglycemia, the glucose infusion $(2.5 \mathrm{mg} / \mathrm{kg}$ per min) is started $1 \mathrm{~min}$ after onset of the insulin infusion. On the basis of the arterial glucose concentrations measured at 2-min intervals, the infusion rate of the glucose pump is adjusted to maintain euglycemia over the full period $(30-35 \mathrm{~min})$ of insulin action. The amount of glucose infused ( $\mathrm{mg} / \mathrm{kg}$ body weight) after insulin administra- tion represents the magnitude of insulin sensitivity and is referred to as the RIST index.

The RIST methodology has numerous advantages as a research and diagnostic tool. It is not affected by pentobarbital anesthesia in rats (4) or isoflurane anesthesia in mice (7). The RIST can be carried out routinely four consecutive times in the anesthetized animal with no significant deterioration (13). The RIST also allows for determination of both the HISS-dependent and HISSindependent insulin action. For example, a RIST carried out in the fed state in Sprague Dawley rats will typically result in a need to administer approximately $230 \mathrm{mg}$ glucose $/ \mathrm{kg}$ body weight in order to prevent the induction of hypoglycemia in response to $50 \mathrm{mU} / \mathrm{kg}$ of insulin. This response consists of both the HISS-dependent and HISS-independent components. Re-testing after hepatic denervation or atropine or inhibition of NOS decreases the RIST index to approximately $100 \mathrm{mg} / \mathrm{kg}$, which represents the HISS-independent component of insulin action. The HISS-dependent component is the component that was eliminated and is calculated from the difference between the control response and the blocked response (Fig. 2). None of the wide variety of stimuli we have studied result in consistent alterations in the HISS-independent component of insulin action except for two disease models described by Macedo's group. The fatty Zucker rat shows equal decreases in both components of insulin action (14) and the spontaneously hypertensive rat shows decreased HISS-dependent action but increased HISS-independent action (15).

The RIST methodology is sufficiently sensitive to allow for detailed dose-response studies. For example, atropine has been shown to result in dose-dependent HDIR (16) with sufficient reproducibility that a submaximal dose of atropine estimated to produce a $75 \%$ block has been used to demonstrate the ability of acetylcholinesterase antagonists to potentiate impaired parasympathetic signaling (17). The dose-dependent production of HDIR with atropine shows dramatic species selectivity with a dose of $3 \mathrm{mg} / \mathrm{kg}$ being required to produce a full blockade in cats compared with a dose of $10 \mu \mathrm{g} / \mathrm{kg}$ in rats.

The pharmacodynamic approach was also used to study the insulin dose-related release of HISS. Doses of insulin that result in a wide range of biological activity $(5-100 \mathrm{mU}$ insulin $/ \mathrm{kg})$ result in a similar proportion of HISS action with approximately $55 \%$ of the glucose disposal effect being accounted for by HISS at all dose ranges. It should be noted, however, that higher doses of insulin appear to result in a protective block of HISS release, which is maintained for at least $2 \mathrm{~h}$ after termination of the high dose of insulin (W.W. Lautt et al., 

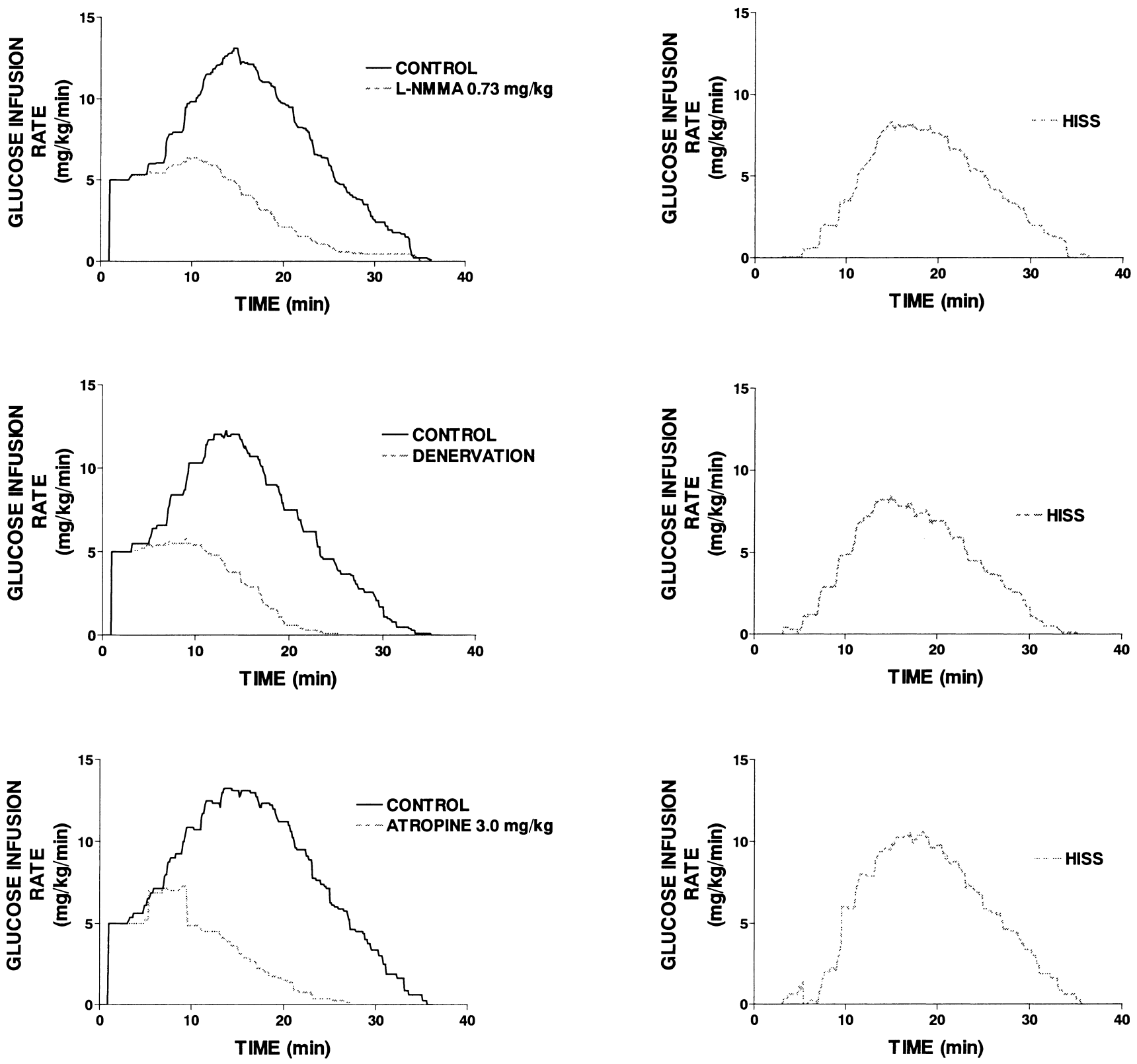

Fig. 2. The mean RIST curves were obtained by averaging glucose infusion rates at 0.1 -min intervals throughout the test after insulin administration from $0-5 \mathrm{~min}(50 \mathrm{mU} / \mathrm{kg})$. Left: Control RIST and the RIST obtained after HISS release blockade by blockade of hepatic nitric oxide synthase (L-NMMA), acute surgical denervation of the liver, or use of atropine to block hepatic muscarinic receptors. Right: HISS-dependent component of insulin action, which is calculated from the difference between the curves on the left. HISS action commenced at $3.1 \pm 0.1 \mathrm{~min}$ after the onset of insulin administration. HISS action continued for 9-10 min after completion of HISS-independent insulin action. Reproduced with permission from the American Journal of Physiology of Gastrointestinal Liver Physiology, Ref. 3.

unpublished). Similarly, at least in the rat, continuous non-pulsatile administration of insulin also results in a blockade of HISS release, thus making the classical 3-h hyperinsulinemic euglycemic clamp method untenable for detection of HISS action in rats (11). There may be a species difference in this regard since HISS action in skeletal muscle in dogs was confirmed to be regulated by cholinergic parasympathetic hepatic control mechanisms using the classical euglycemic clamp (6).

The RIST methodology is also sufficiently precise to permit demonstration of the liver as the drug target through the development and reversal of HDIR by comparing effects of drugs administered directly to the liver through the portal vein with the same dose 
administered systemically. For example, a sub-maximal dose of a NOS inhibitor delivered into the portal vein resulted in insulin resistance that was not produced when the drug was administered intravenously. If the drug had a significant direct action on skeletal muscle, the intravenous response would have been at least as large or larger than the response seen with administration into the portal vein. Similarly, when HDIR was produced by surgical denervation or administration of a NOS inhibitor, reversal of HDIR with NO donors through the systemic venous or portal venous site also confirmed that the site of restoration was dependent upon NO actions in the liver. Similar approaches have been used to show that atropine targets the liver (16) as do COX inhibitors (18) to produce HDIR.

\section{Control of HISS release}

The first observation of hepatic nerve-dependent insulin action was a serendipitous discovery made while carrying out an experiment to determine if insulin was capable of activating receptors in the brain to affect glucose metabolism through hepatic nerves. The experiment resulted in the observation that the hypoglycemic response to a bolus of insulin was decreased following hepatic denervation. Further studies demonstrated that the regulatory mechanism was dependent upon hepatic parasympathetic nerves $(5,19)$. Subsequently we determined that the glucose disposal effects, although determined by hepatic signaling mechanisms, were occurring in skeletal muscle (10). This observation has been elegantly confirmed using the conscious dog model (6).

After demonstrating that the parasympathetic nerves in the liver were controlling HISS action, we then tested the hypothesis, based upon parasympathetic functions in other tissues, that $\mathrm{NO}$ generation was also part of the signaling pathway. The RIST methodology allowed demonstration of the role of $\mathrm{NO}$ in the signaling pathway $(20,21)$. Recent studies from Macedo's group (M.P. Macedo, personal communication) have shown that the sequential signaling involves cholinergic activation of the NOS pathway and that NO acts through guanylyl cyclase (22) and hepatic glutathione (23). NO has also been shown to regulate insulin sensitivity in rabbits (24). Furthermore, the observation that NO-dependent mechanisms are often also regulated by the prostaglandin pathway suggested the hypothesis that prostaglandins may also have a role in regulating HISS. We demonstrated that COX inhibition in the liver was also capable of blocking HISS production (18). Precisely how the NO or prostaglandins are involved with producing HISS is currently unclear, but blockade of guanylyl cyclase to produce HDIR suggests that NO is acting through the classical guanylyl cyclase pathway. The hepatic glutathione level, which is reduced by fasting and restored by feeding, is also a determinant of HISS production by unknown mechanisms (23).

Although it is clear that feeding results in full activation of the MIS process, the precise signal is unclear. An apparently paradoxical comparison between the responses in fed conscious versus anesthetized rats may provide some clue as to the signaling mechanism. When rats are fasted and re-fed and then anesthetized and subjected to a RIST, a full MIS is demonstrated. The RIST index obtained can be reproduced four times over an 8-h period with no significant decrease seen (13). In contrast, a fasted rat that is then re-fed and tested in the conscious state using standard RIST methodology shows a RIST index and MIS that is similar to that seen in the anesthetized animal. However, subsequent tests in the conscious state without further feeding result in a progressive decline in the RIST index that is similar to that seen in previous studies examining the effect of duration of fasting (4). Anesthesia results in paralysis of gastrointestinal movement so that the bolus of food in the stomach of the fed, anesthetized rat appears unaltered in composition or content at the end of the $8-10$-h period of testing in the anesthetized animal. These data may suggest that something as simple as gastric expansion may be part of the afferent signal leading to parasympathetic tone regulation in the liver. It should be stressed, however, that simply mimicking the parasympathetic signal with acetylcholine in the fasted rat does not result in a feeding signal. Furthermore, fed rats with reduced hepatic glutathione levels produced by 1-buthionine-sulfoxamine (23) also show complete HDIR even when provided with exogenous $\mathrm{NO}$, thus suggesting that there are at least two sequential elements involved with the full signaling process. Recent data also suggest that the afferent signal may also derive from the liver since sensory denervation of the liver using topical capsaicin is reported to result in HDIR (24). The studies of Porszasz et al. (24) indicating that destruction of the hepatic afferent nerves leads to insulin resistance could suggest that the feeding signal is derived from sensory input from the liver; however, it is not clear if the insulin resistance reported represents HDIR as the studies were carried out in the fasted state. The source and stimulus for the sensory limb of the permissive reflex represents an important focus of research.

\section{Role of HDIR in pathology}

As described in the overview, the HISS hypothesis is compatible with the suggestion that HDIR accounts for 
postprandial hyperglycemia, hyperinsulinemia, hyperlipidemia, and results in a state of metabolic obesity where nutrients are directed towards energy storage in the form of lipids rather than glycogen stores in skeletal muscle. Insulin resistance is accounted for by HDIR in a wide variety of models including the spontaneously hypertensive rat (15), sucrose fed rats $(25,26)$, animals with liver disease induced by chronic bile duct ligation (27) or chemical toxins (Z. Ming and W.W. Lautt, unpublished observations), adult offspring of fetal alcohol exposure $(28,29)$, acute stress (L. Seredycz and W.W. Lautt, unpublished observation), ageing (M.P. Macedo, personal communication), physical interruption of hepatic parasympathetic nerves $(1,6,30)$, pharmacological blockade of hepatic muscarinic cholinergic receptors $(5,19)$ or NO production $(20,21,31)$ or COX (18), and blockade of hepatic guanylyl cyclase (22) or depletion of hepatic glutathione (23). In all of these models, insulin resistance is accounted for by HDIR.

The current paradigm for insulin resistance focuses on peripheral defects in insulin signaling with the majority of studies being carried out in the fasted state. The focus on the fasting state appears misdirected. It has been suggested that hyperglycemia-induced overproduction of superoxide by the mitochondrial electrontransport chain accounts for the four main molecular mechanisms implicated in glucose-mediated vascular damage associated with blindness, renal failure, nerve damage, atherosclerosis, stroke, and hindlimb amputation (32). The importance of the post-meal, rather than the fasting, metabolic status is amply demonstrated in a number of recent studies.

The relationship between glycosylated hemoglobin $\left(\mathrm{HbA}_{1 \mathrm{c}}\right)$ and plasma glucose in patients with type 2 diabetes was determined at four time points during the day and found to be significantly predicted by plasma glucose levels measured only at post-lunch and extended post-lunch $(5 \mathrm{~h})$ time points (33). The strongest ageand sex-adjusted relative risk for all-cause and cardiovascular mortality was associated with 2 -h post-load plasma glucose levels (34). Increased mortality risk has been associated with 2-h post-load plasma glucose levels to a much greater extent than with fasting plasma glucose $(35,36)$. Isolated post-load hyperglycemia is a strong predictor of mortality $(35,37-41)$. Loss of post-meal glycemic control can account for the postprandial hyperglycemia and elevated insulin secretion known to occur in early stages of type 2 diabetes; the explanation for the post-meal hyperglycemia has generally been based upon observations suggesting that first phase insulin secretion is impaired to varying degrees (reviewed by 42).
The approach of screening for type 2 diabetes using the fasted metabolic status, while convenient, is not effective. In a recent review evaluating the status of screening for type 2 diabetes, Engelgau et al. (37) stated that one of the criteria for appropriate screening is that the tests should detect the preclinical stage of disease and that the tests be shown to be acceptable and reliable. The conclusion that current screening recommendations are not consistent with available evidence was briefly reviewed. Evidence is accumulating that most people with a $54-67 \%$ range of impaired glucose tolerance have fasting glucose in the normal range. Pooled analysis of 20 different European studies showed as many as $31 \%$ who were diabetic according to postchallenge plasma glucose but had normal fasting values and therefore would not have been detected by a screening procedure based on fasting glucose measurements.

The discovery of HISS-dependent insulin action and MIS is consistent with recent perceptions that the current paradigm of insulin resistance has sufficient anomalies to require a re-examination and increased focus on the postprandial nutrition partitioning.

\section{Therapeutic potential}

In a recent review evaluating new drug targets for type 2 diabetes and the metabolic syndrome, Moller (43) emphasized that current therapies for type 2 diabetes were developed in the absence of defined molecular targets or an understanding of disease pathogenesis: "These therapies have limited efficacy, limited tolerability, and significant mechanism-based side effects. Of particular concern is the tendency for most treatments to enhance weight gain. Several current approaches are also associated with episodes of hypoglycemia, and few of the available therapies adequately address underlying defects such as obesity and/or insulin resistance." $\mathrm{He}$ further concluded that "Thus, newer approaches are definitely needed. Particular emphasis should be placed on finding and using mechanisms that are dependent on physiological responses and that result in weight loss". With the current understanding of the physiology and pathology related to HISS, it appears that targeting of this mechanism offers a novel approach to restoring normal physiological insulin sensitivity in skeletal muscle where the insulin resistance is generally acknowledged to primarily occur in many insulin resistant states.

One approach we are currently exploring is to mimic the hepatic parasympathetic feeding signal by the use of cholinergic agonists or NO donors so that the permissive signal will restore the ability of a pulse of insulin to release a pulse of HISS. This approach has been 
successfully demonstrated in the acute model of insulin resistance produced by surgical denervation of the liver in rats (2) and has been recently confirmed in dogs (6). Insulin resistance produced by HDIR in a chronic liver disease model was also completely reversible by the provision of a cholinergic agonist directly to the liver (27). Another approach we have recently demonstrated to be effective depends upon the ability to potentiate existing but weak parasympathetic signals through the use of acetylcholinesterase inhibitors to potentiate the biological response to endogenously released acetylcholine (17) and the use of phosphodiesterase inhibitors to potentiate the biological response to hepatic NO production (W.W. Lautt et al., unpublished observations). In some models it appears necessary not only to mimic or potentiate the parasympathetic signal but also to restore hepatic glutathione to levels normally produced by feeding (M.P. Macedo and W.W. Lautt, unpublished observations).

\section{Future direction}

The discovery of the MIS process and confirmation of many aspects of the HISS hypothesis suggest the need for a paradigm shift in research. In addition, this new paradigm in the understanding of insulin resistance gives rise to new therapeutic pathways and links. Early quantifiable diagnostics, targeted mechanistic-based therapeutics, and understanding of the effect of lifestyle and drugs on the MIS process is predicted to have a major impact on the epidemics of diabetes and obesity and their critical interrelatedness.

\section{Acknowledgments}

Many trainees, support staff, and collaborators have been instrumental in these studies, most notably, chronologically, Hongsheng Xie, Dallas Legare, Parissa Sadri, Maria Reid, and Paula Macedo. Graduate students and technologists primarily in the New University of Lisbon and the University of Manitoba continue to advance the discovery process. Manuscript preparation was greatly assisted by Karen Sanders. Conflict of interest declaration: the author is affiliated with DiaMedica Inc. (Winnipeg, Manitoba, Canada), the licensor of technology to diagnose and treat insulin resistance through the HISS pathway.

\section{References}

1 Xie H, Tsybenko VA, Johnson MV, Lautt WW. Insulin resistance of glucose response produced by hepatic denervations. Can J Physiol Pharmacol. 1993;71:175-178.
2 Xie H, Lautt WW. Insulin resistance caused by hepatic cholinergic interruption and reversed by acetylcholine administration. Am J Physiol. 1996;271:E587-E592.

3 Lautt WW, Macedo MP, Sadri P, Takayama S, Ramos FD, Legare DJ. Hepatic parasympathetic nerve-dependent control of peripheral insulin sensitivity is determined by feeding and fasting: dynamic control of HISS-dependent insulin action. Am J Physiol Gastrointest Liver Physiol. 2001;281:G29-G36.

4 Latour MG, Lautt WW. Insulin sensitivity regulated by feeding in the conscious unrestrained rat. Can J Physiol Pharmacol. 2002;80:8-12.

5 Xie H, Lautt WW. Induction of insulin resistance by cholinergic blockade with atropine in the cat. J Auton Pharmacol. 1995; 15:361-369.

6 Moore MC, Satake S, Baranowski B, Hsieh P-S, Neal DW, Cherrington AD. Effect of hepatic denervation on peripheral insulin sensitivity in conscious dogs. Am J Physiol Endocrinol Metab. 2002;282:E286-E296.

7 Latour MG, Chan CC. A rapid insulin sensitivity test (RIST) in anesthetized mice. Diabetes. 2002;51 Suppl 2:A422.

8 Lautt WW. State of the Art 1997: Hepatic parasympathetic nerves and glucose metabolism. In: Haussinger D, Jungermann K, editors. Liver and nervous system. Falk Symposium No. 103. UK: Kluwer Academic Publishers; 1998. p 1-14.

9 Lautt WW. Practice and principles of pharmacodynamic determination of HISS-dependent and HISS-independent insulin action: methods to quantitate mechanisms of insulin resistance. Med Res Rev. 2003;23:1-14.

10 Xie H, Lautt WW. Insulin resistance of skeletal muscle produced by hepatic parasympathetic interruption. Am J Physiol. 1996; 270:E858-E863.

11 Reid MAG, Latour MG, Legare DJ, Rong N, Lautt WW. Comparison of the rapid insulin sensitivity test (RIST), the insulin tolerance test (ITT), and the hyperinsulinemic euglycemic clamp (HIEC) to measure insulin action in rats. Can J Physiol Pharmacol. 2002;80:811-818.

12 Xie H, Zhu L, Zhang YL, Legare DJ, Lautt WW. Insulin sensitivity tested with a modified euglycemic technique in cats and rats. J Pharmacol Toxicol Meth. 1996;35:77-82.

13 Lautt WW, Wang X, Sadri P, Legare DJ, Macedo MP. Rapid insulin sensitivity test (RIST). Can J Physiol Pharmacol. 1998; 76:1080-1086.

14 Ribeiro RT, Duarte-Ramos F, Macedo MP. The fatty Zucker $\mathrm{fa} / \mathrm{fa}$ rat show a dysfunction of the HISS dependent and independent components of insulin action. Proc West Pharmacol Soc. 2001;44:27-28.

15 Ribeiro RT, Duarte-Ramos F, Macedo MP. Effect of the hepatic insulin sensitizing substance (HISS) action in the spontaneously hypertensive rats. Proc West Pharmacol Soc. 2001;44:29-30.

16 Takayama S, Legare DJ, Lautt WW. Dose-related atropineinduced insulin resistance: comparing intraportal versus intravenous administration. Proc West Pharmacol Soc. 2000;43:3334.

17 d'Almeida MS, Reid MAG, Legare DJ, Lautt WW. Inhibition of acetylcholinesterase: a novel approach to improve insulin sensitivity in experimental models of insulin resistance. Can J Diabetes. 2003;27:332.

18 Sadri P, Lautt WW. Insulin resistance caused by hepatic COX inhibition. Diabetes. 2000;49 Suppl 1:A245.

19 Xie H, Lautt WW. Insulin resistance produced by hepatic 
denervation or muscarinic cholinergic blockade. Proc West Pharmacol Soc. 1994;37:39-40.

20 Sadri P, Legare DJ, Lautt WW. Insulin resistance caused by nitric oxide synthase inhibition. Proc West Pharmacol Soc. 1997;40:19-20.

21 Sadri P, Lautt WW. Blockade of hepatic nitric oxide synthase causes insulin resistance. Am J Physiol. 1999;277:G101-G108.

22 Correia NC, Guarino MP, Raposo J, Macedo MP. Hepatic guanylyl cyclase inhibition induces HISS dependent insulin resistance. Proc West Pharmacol Soc. 2002;45:57-58.

23 Guarino MP, Afonso RA, Raimundo N, Raposo JF, Macedo MP. Hepatic glutathione and nitric oxide are critical for hepatic insulin-sensitizing substance action. Am J Physiol Gastrointest Liver Physiol. 2003;284:G588-G594.

24 Porszasz R, Legvari G, Nemeth J, Literati PN, Szolcsanyi J, Szilvassy $Z$. The sensory nitrergic nature of the hepatic insulin sensitizing substance mechanism in conscious rabbits. Eur $\mathrm{J}$ Pharmacol. 2002;443:211-212.

25 Ribeiro RT, Duarte-Ramos F, Macedo MP. The hepatic insulin sensitizing substance (HISS) action is decreased in rats on a high-sucrose diet. Proc West Pharmacol Soc. 2001;44:31-32.

26 d'Almeida MS, Legare DJ, Lautt WW. Decreased hepatic insulin sensitizing substance (HISS) accounts for insulin resistance observed in sucrose fed rats. Can J Diabetes. 2002;2:301.

27 Lautt WW, Xie H. Intraportal acetylcholine reverses insulin resistance caused by chronic bile duct ligation. Proc West Pharmacol Soc. 1998;41:35-36.

28 Minuk GY, Meyers AFA, Legare DJ, Sadri P, Lautt WW. Fetal exposure to alcohol results in adult insulin resistance in the rat. Proc West Pharmacol Soc. 1998;41:39-40.

29 Sadri P, Legare DJ, Takayama S, Lautt WW. Fetal ethanol exposure causes hepatic insulin sensitizing substance-dependent insulin resistance. Can J Diabetes. 2003;27:239-247.

30 Latour MG, Lautt WW. The hepatic vagus nerve in the control of insulin sensitivity in the rat. Auton Neurosci. 2002;95:25130.

31 Guarino MP, Correia NC, Raposo JF, Macedo MP. Nitric oxide synthase inhibition decreases hepatic insulin sensitizing sub- stance output which is reversed by SIN-1 but not by nitroprusside. Proc West Pharmacol Soc. 2001;44:25-26.

32 Brownlee M. Biochemistry and molecular cell biology of diabetic complications. Nature. 2001;414:813-819.

33 Avignon A, Radauceanu A, Monnier L. Nonfasting plasma glucose is a better marker of diabetic control than fasting plasma glucose in type 2 diabetes. Diabetes Care. 1997;20:1822-1826.

34 de Vegt F, Dekker JM, Ruhé HG, Stehouwer CDA, Nijpels GBLM, Heine RJ. Hyperglycaemia is associated with all-cause and cardiovascular mortality in the Hoorn population: the Hoorn study. Diabetologia. 1999;42:926-931.

35 DECODE Study Group, on behalf of the European Diabetes Epidemiology Group. Glucose tolerance and mortality: comparison of WHO and American Diabetes Association diagnostic criteria. Lancet. 1999;354:617-621.

36 Hanefeld M, Fischer S, Julius U, et al. Risk factors for myocardial infarction and death in newly detected NIDDM: the Diabetes Intervention Study, 11-year follow-up. Diabetologia. 1996;39:1577-1583.

37 Engelgau MM, Narayan KMV, Herman WH. Screening for type 2 diabetes. Diabetes Care. 2000;23:1563-1580.

38 Vaccaro O, Ruffa G, Imperatore G, Iovino V, Rivellese AA, Riccardi G. Risk of diabetes in the new diagnostic category of impaired fasting glucose. Diabetes Care. 1999;22:1490-1493.

39 Shaw JE, Hodge AM, de Courten M, Chitson P, Zimmet PZ. Isolated post-challenge hyperglycemia confirmed as a risk factor for mortality. Diabetologia. 1999;42:1050-1054.

40 Simon C, Brandenberger G. Ultradian oscillations of insulin secretion in humans. Diabetes. 2002;51 Suppl 1:S258-S261.

41 Simon C, Follenius M, Brandenberger G. Postprandial oscillations of plasma glucose insulin and C-peptide in man. Diabetologia. 1987;30:769-773.

42 Del Prato S, Tiengo A. The importance of first-phase insulin secretion: implications for the therapy of type 2 diabetes mellitus. Diabetes Metab Res Rev. 2001;17:164-174.

43 Moller DE. New drug targets for type 2 diabetes and the metabolic syndrome. Nature. 2001;414:821-827. 